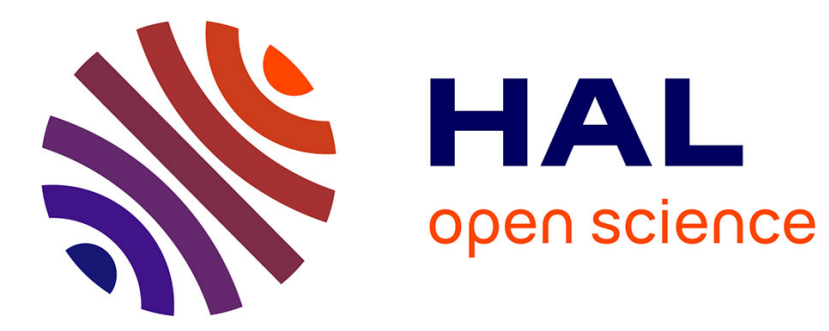

\title{
Accurate LoRa Performance evaluation using Marcum function
}

\author{
Jules Courjault, Baptiste Vrigneau, Matthieu Gautier, Olivier Berder
}

\section{To cite this version:}

Jules Courjault, Baptiste Vrigneau, Matthieu Gautier, Olivier Berder. Accurate LoRa Performance evaluation using Marcum function. IEEE International conference on Global Communications (Globecom), Dec 2019, Hawaï, United States. hal-02418665

\section{HAL Id: hal-02418665 https://hal.science/hal-02418665}

Submitted on 19 Dec 2019

HAL is a multi-disciplinary open access archive for the deposit and dissemination of scientific research documents, whether they are published or not. The documents may come from teaching and research institutions in France or abroad, or from public or private research centers.
L'archive ouverte pluridisciplinaire HAL, est destinée au dépôt et à la diffusion de documents scientifiques de niveau recherche, publiés ou non, émanant des établissements d'enseignement et de recherche français ou étrangers, des laboratoires publics ou privés. 


\section{Accurate LoRa Performance evaluation using Marcum function}

\author{
Jules Courjault \\ Univ Rennes, CNRS, IRISA \\ Lannion, France
}

\author{
Baptiste Vrigneau \\ Univ Rennes, CNRS, IRISA \\ Lannion, France
}

\author{
Matthieu Gautier \\ Univ Rennes, CNRS, IRISA \\ Lannion, France
}

\author{
Olivier Berder \\ Univ Rennes, CNRS, IRISA \\ Lannion, France
}

\begin{abstract}
In the last years, Internet of Things (IoT) grew up in an exponential behavior and required long range and low power wireless transmissions. Several standards were proposed and LoRa has emerged as a high potential candidate for many IoT solutions. LoRa modulation is based on a chirp spread-spectrum technique and offers efficient transmission up to $50 \mathrm{kbps}$ over several kilometers. Although the principle is known and studied for decades now, the performance in terms of symbol or bit error probability has been theoretically analyzed in few papers only. Closed-form approximations for additive white Gaussian noise and Rayleigh fading were recently proposed. In this paper, we propose a new approach based on Marcum function. Simulations and comparisons with the state of the art show that the proposed approximation of the Binary Error Probability is up to ten times more accurate for a full SNR range.
\end{abstract}

Index Terms-LP-WAN, LoRa transmission, Binary Error Probability performance analysis, Marcum function

\section{INTRODUCTION}

During the last decade, the number of small connected nodes grew up exponentially for various applications such as environmental monitoring or connected farms [1]. Many emerging solutions recently arised to cover the needs for long range and very low energy consumption wireless transmissions. A panel of standards for Low Power Wide Area Network (LP-WAN) was presented in the paper [2]. Among the candidates, LoRa is a long range communication technology promoted by the LoRa Alliance. The LoRa physical layer, which allows long-range, low-power and low data rate communications, is developed by Semtech [3] [4]. LoRa communications can use frequency bands of $433 \mathrm{MHz}, 868 \mathrm{MHz}$ or $915 \mathrm{MHz}$ and its data rate can reach up to $50 \mathrm{kbps}$. It uses the Chirp Spread Spectrum (CSS) modulation with a linear variation of frequency on time [5] [6], which allows the reduction of both interference and Doppler effects [7]. LoRa modulation can be configured with three parameters: the bandwidth $B W$, the spreading factor $S F$, and the coding ratio $C R$. Although LoRa is described in the patent, rigorous theoretical studies of this technology are still missing. The author in [8] proposed to rigorously study the modulation and demodulation in a mathematical approach but, as highlighted in [9], the paper still lacks a theoretical analysis of the Bit Error Probability (BEP). As explained later in the present paper, the problem is less to find the theoretical expression, it was already done in [10], than an approximation easily computable. All proposed expressions in the literature are based on the complementary error function, while we propose an accurate approximation of the BEP for an Additive White Gaussian Noise (AWGN) channel based on the Marcum function [11] which was already employed in communications theory [12].

The rest of the paper is organized as follows: Section II presents the required basics and the state of the art of LoRa BER approximations. Section III introduces the novelty based on a Taylor expansion and the main results with Marcum function. Section IV compares proposed results to the state of the art before the conclusion in Section V.

\section{STATE OF THE ART}

\section{A. LoRa BEP theoretical expression and issues}

For conciseness, only the needed equations are introduced and the reader can refer to [8] and [9] for detailed explanations. We remind that the receiver first applies a down chirp, secondly performs the discrete Fourier transform of the receive signal, and third estimates the symbols with the maximum value criterion. As the symbols have the same probability and the problem is equivalent to a permutation, the study can focus on the probability of correct decision of the symbol $m$, denoted $\overline{\mathbb{P}}_{m}$. According to [9], [10], this probability can be expressed as follows:

$$
\overline{\mathbb{P}}_{m}=F_{\chi 2}\left(\frac{\left|\sqrt{N}+W_{p}[N-m]\right|^{2}}{\sigma^{2}}\right)^{N-1},
$$

where $N=2^{S F}, S F$ is the spreading factor and is an integer between 7 to 12 , and $W_{p}[N-m]$ is a Gaussian complex noise of variance $\sigma^{2}$. By using the cumulative density function (cdf) of a $\chi^{2}$ random with 2 degrees of freedom $F_{\chi 2}(x)=$ $1-\exp \left(-\frac{x}{\sigma^{2}}\right)$, eq.(1) can be written:

$$
\overline{\mathbb{P}}_{m}=\left(1-\exp \left(\frac{\left|\sqrt{N}+W_{p}[N-m]\right|^{2}}{\sigma^{2}}\right)\right)^{N-1} .
$$

By defining the Signal-to-Noise Ratio SNR $=1 / \sigma^{2}$ and the random variable

$$
Z=\frac{\left|\sqrt{N}+W_{p}[N-m]\right|^{2}}{\sigma^{2}},
$$

$Z$ follows a non central $\chi^{2}$ law with 2 degrees of freedom and the noncentrality parameter $\lambda=2 N$. SNR. Its probability density function (pdf) is expressed as:

$$
f_{Z}(x)=\frac{1}{2} \exp \left(-\frac{x+\lambda}{2}\right) I_{0}(\sqrt{\lambda x}),
$$


with $I_{0}($.$) the modified Bessel function of the second kind of$ order 0. Finally, the last step is the computation of the average Symbol Error Probability (SEP):

$$
\begin{aligned}
\mathrm{SEP} & =\int_{0}^{+\infty}\left(1-\left(1-e^{-z / 2}\right)^{N-1}\right) f_{Z}(z) d z, \\
& =\frac{1}{N} \sum_{k=1}^{N-1} C_{N}^{k+1}(-1)^{k+1} e^{-N \cdot \operatorname{SNR} \frac{k}{(k+1)}}
\end{aligned}
$$

with $C_{N}^{k+1}=N ! /(k+1) !(N-k-1)$ ! the binomial coefficient. However, the numerical computation of this result suffers from accuracy issue and the results can not be obtained when $S F$ is greater than 6 . This problem was already pointed out in [9], [10], and justifies the crucial need for finding accurate approximations. Generally, the performance is expressed with the BEP and due to equiprobability of the wrong symbol, the following relation can be used:

$$
\mathrm{BEP}=\frac{2^{S F-1}}{2^{S F}-1} \mathrm{SEP} .
$$

\section{B. Existing approximations of the BEP}

Reynders et al. proposed in [13] the approximation:

$$
\mathrm{BEP} \approx Q\left(\frac{\log _{12}(S F)}{\sqrt{2}} \mathrm{SNR}\right),
$$

with $Q(x)$ the Q-function or the tail distribution of the standard normal distribution. Another study [6] gives the following expression that relies on numerical fitting:

$$
\mathrm{BEP} \approx 0.5 \cdot Q(1.28 \sqrt{S F . \mathrm{SNR}}-1.28 \sqrt{S F}+0.4) .
$$

Recently, the authors in [9] proposed two new accurate estimations of the BEP for LoRa communications in AWGN channel and a Rayleigh channel. The principle is to approximate a Ricean distribution by a Gaussian one and the expression for AWGN channel is:

$$
\mathrm{BEP} \approx 0.5 \cdot Q\left(\frac{\sqrt{N \cdot \mathrm{SNR}}-\left(H_{N-1}^{2}-\frac{\pi^{2}}{12}\right)^{1 / 4}}{\sqrt{H_{N-1}-\sqrt{H_{N-1}^{2}-\frac{\pi^{2}}{12}}+0.5}}\right),
$$

where $H_{m}$ is the $m^{t h}$ harmonic number which can be approximated by $H_{m} \approx \log (m)+\frac{1}{2 m}+0.57722$. The second approximation is obtained from the former with some simplifications and approximations:

$$
\mathrm{BEP} \approx 0.5 \cdot Q(\sqrt{2 N \cdot \mathrm{SNR}}-\sqrt{1.386 \cdot S F+1.154}) .
$$

\section{Closed-FORM USing MARCUM FUnCTION}

\section{A. Approximation of $\overline{\mathbb{P}}_{m}$}

As introduced above, the previous works focused on approximating Chi squared or Ricean distributions with a Gaussian one. The novelty of this work is to find an approximation of $g(z)=1-\left(1-e^{-z / 2}\right)^{N-1}$ while keeping the non-central Chi squared distribution. When $g(z)$ is extended in a sum, the term $e^{-k z / 2}$ is less significant when $k$ and $z$ increase. Thus, the sum can be limited to $\epsilon$ terms. It is equivalent to find the

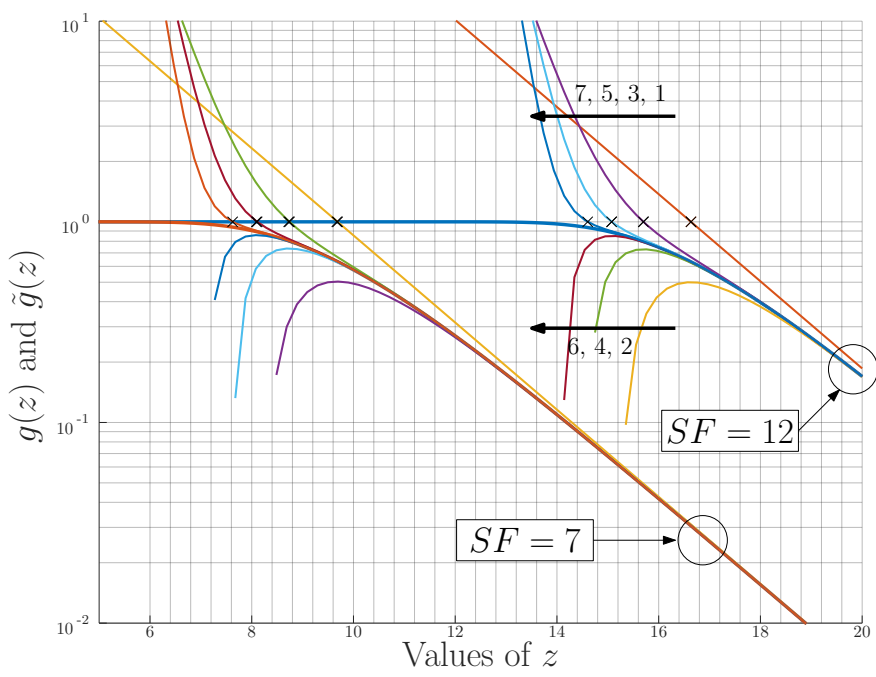

Fig. 1: Comparison of the exact function $g(z)$ and approximations with different orders $\epsilon$ from 1 to 7 and $S F=7,12$. Values of $z_{c}$ are plotted with cross marker.

approximation thanks to the Taylor's series for high values of $z$ and the first major expression is obtained:

$$
g(z) \approx \tilde{g}(z)=\sum_{k=1}^{\epsilon}(-1)^{k+1} C_{N-1}^{k} e^{-k z / 2} .
$$

Fig. 1 shows the comparison with the exact $g(z)$ and different approximation orders, $\epsilon=1$ to 7 . The good point is that binomial coefficients are limited to low values of $k$, which solves a computational issue. Since $N$ is high, especially for $S F=12$, the binomial coefficient $C_{N-1}^{k}$ will suffer from loss of precision, typically when $k$ is more than 5. Although the approximation is tightly close for high values of $z$, the divergence is problematic: the approximation tends toward infinity for odd values of $\epsilon$ and toward zero for even orders. Nevertheless, a simple and efficient approximation based on a piecewise definition is obvious in order to obtain the initial value 1:

$$
g(z) \simeq\left\{\begin{array}{cc}
1 & \text { if } z \leq z_{c} \\
\tilde{g}(z) & \text { if } z>z_{c}
\end{array}\right.
$$

where $z_{c}$ is a threshold depending on the values of $\epsilon$ and $S F$ that will be discussed in the next paragraph. The different values of $z_{c}$ are available in the Fig. 1 and a quick observation is that the piecewise approximation should be accurate for low values of $\epsilon$ (inferior to 7).

The next step is to compute the average as in eq.(5):

$$
\begin{aligned}
\mathrm{SEP}= & \int_{0}^{z_{c}} f_{Z}(z) d z+ \\
& \int_{z_{c}}^{\infty} \sum_{k=1}^{\epsilon}(-1)^{k+1} C_{N-1}^{k} e^{-k z / 2} f_{Z}(z) d z .
\end{aligned}
$$

By using the definition of the pdf in (4) and the variable changes $x_{0}=\sqrt{z}$ and $x_{k}=\sqrt{z(k+1)}$, the SEP can be 


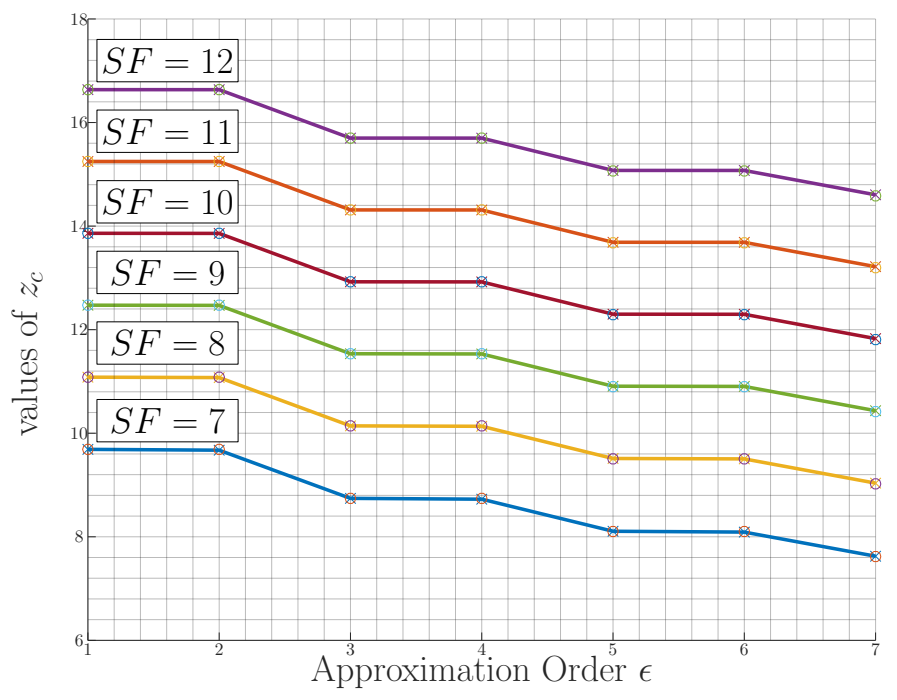

Fig. 2: Approximation of the threshold $z_{c}$ : numerical solutions are plotted with crosses and the approximations with circles.

expressed by this practical result after some mathematical manipulations:

$$
\begin{array}{r}
\mathrm{SEP}=1+ \\
\sum_{k=1}^{\epsilon+1} \frac{C_{N}^{k}}{N}(-1)^{k} e^{-\frac{N \cdot \mathrm{SNR}(k-1)}{k}} Q_{1}\left(\sqrt{\frac{2 N}{k} \mathrm{SNR}}, \sqrt{k z_{c}}\right),
\end{array}
$$

with $Q_{1}$ the Marcum function of order 1 defined by:

$$
Q_{M}(a, b)=\int_{b}^{\infty} x\left(\frac{x}{a}\right)^{M-1} e^{-\frac{x^{2}+a^{2}}{2}} I_{M-1}(a x) d x .
$$

\section{B. How to find $z_{c}$ ?}

Almost all parameters are known for computing (15) except the value of $z_{c}$. The problem is not trivial and we propose a solution for low values of the order $\epsilon$. One should note that the results in the next section and the above remark confirm that the order does not need to be greater than 7. First, by applying the variable change $\zeta=e^{-\frac{z}{2}}$ in (12), solving $\tilde{g}(z)=1$ is equivalent to find the roots of a $\epsilon$-order polynom. Since the approximation with even values of $\epsilon$ can not reach the value 1 , the study is limited to odd value cases. The only known assumption is that $z_{c}$ is the unique positive real root. However, we are able to obtain the exact solutions for $\epsilon=1$ and $\epsilon=3$. By using the notation $\zeta_{c}(\epsilon)=e^{-\frac{z_{c}(\epsilon)}{2}}$, we have:

$$
\begin{array}{rlrl}
\zeta_{c}(1) & = & 1 /(N-1) \\
\zeta_{c}(3) & = & \tau-\frac{N-4}{(N-2)(N-3)^{2}} \frac{1}{\tau}+\frac{1}{N-3} \\
\tau & =\left(\frac{(N-4)(N-5)}{(N-1)(N-2)(N-3)^{3}}+\frac{\sqrt{2}(N-4)}{(N-1)(N-2)^{1.5}(N-3)^{1.5}}\right)^{\frac{1}{3}} \\
\tau & \simeq & \frac{(1+\sqrt{2})^{\frac{1}{3}}}{N}
\end{array}
$$

The last simplification for $\tau$ is obtained when $N$ is larger than 1. A straightforward numerical observation leads to a heuristic polynomial function of order 1 that gives the exact

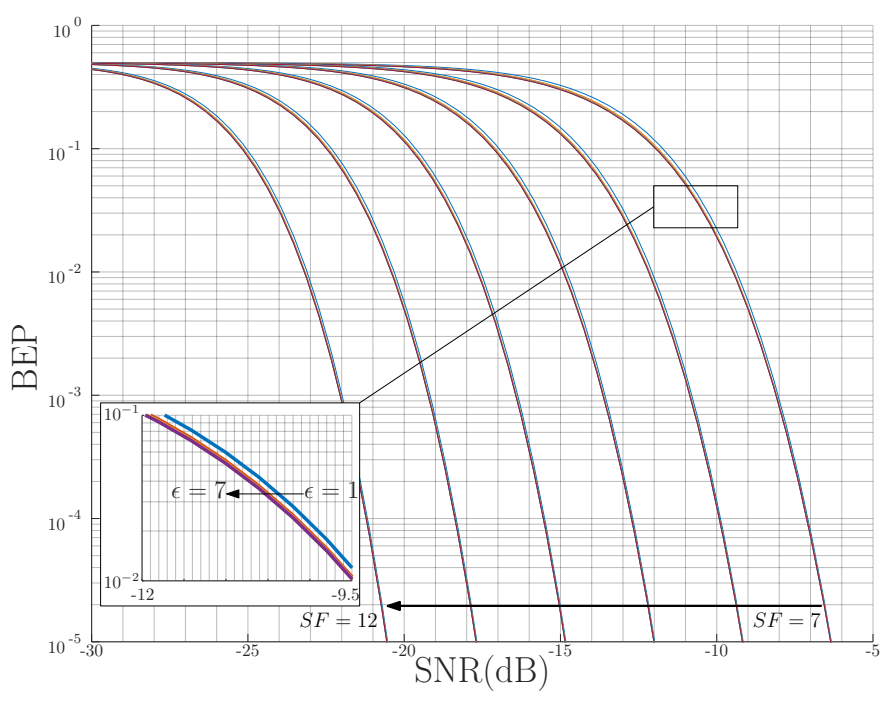

Fig. 3: BEP approximation for order $\epsilon=1$ to 7 and $S F=7$ to 12 .

values of $\zeta_{c}$ and $z_{c}$ for $\epsilon=1,3$ and an approximation for higher values:

$$
\begin{array}{r}
\zeta_{c}(\epsilon)=\alpha_{1} \epsilon+\alpha_{0} \\
\alpha_{1}=\left(\zeta_{c}(3)-\zeta_{c}(1)\right) / 2, \alpha_{0}=\zeta_{c}(1)-\alpha_{1}
\end{array}
$$

Concerning the even cases, we propose to define $z_{c}$ as the value maximizing $\tilde{g}(z)$, i.e. $z_{c}$ is the solution to the equation $\frac{\partial \tilde{g}(z)}{\partial z}=0$. The result has also a polynomial form and the same study for odd cases can be applied. However, for conciseness, we proposed the heuristic approximation $z_{c}(\epsilon)=z_{c}(\epsilon-1)$ when $\epsilon$ is even, as shown on Fig. 2. Fig. 2 shows the comparison between a numerical solving of $z_{c}$ and the proposed approximation. We can observe that the approximation is still tight to the numerical value for $\epsilon=5,7$.

\section{RESULTS AND COMPARISONS}

\section{A. Impact and choice of the order $\epsilon$}

The BEP approximation is illustrated by Fig. 3 for different values of the order $\epsilon=1$ to 7 and $S F=7$ to 12 . It is obvious that the approximation converges very fast: if the order $\epsilon=1$ is still visible, the other curves are mingled. A quick conclusion is that the order $\epsilon=1$ is already a good approximation with the best trade-off between complexity and accuracy, and it is not necessary to increase $\epsilon$ higher than 3 . In order to confirm this, a convergence parameter is defined by:

$$
\Delta_{\mathrm{BEP}, i \rightarrow j}=\left(\left.\mathrm{BEP}\right|_{\epsilon=i}-\left.\mathrm{BEP}\right|_{\epsilon=j}\right) /\left.\mathrm{BEP}\right|_{\epsilon=i} .
$$

It allows the evaluation of the BEP variation when the order $\epsilon$ is increased. Fig. 4 shows three cases $\Delta_{\mathrm{BEP}, 1 \rightarrow 3}, \Delta_{\mathrm{BEP}, 3 \rightarrow 5}$, and $\Delta_{\mathrm{BEP}, 5 \rightarrow 7}$ as a function of SNR for different values of $S F$. The variation when the order $\epsilon$ increases from 1 to 3 is inferior to $10 \%$, the variation is lower than $3 \%$ when the order $\epsilon$ changes from 3 to 5 , and less that $1 \%$ for the next step 5 to 7. Moreover, Table I shows maximum values of $\Delta_{\mathrm{BEP}, i \rightarrow i+1}$ for $i=1$ to 6 . Since the approximation for even $\epsilon$ is less 

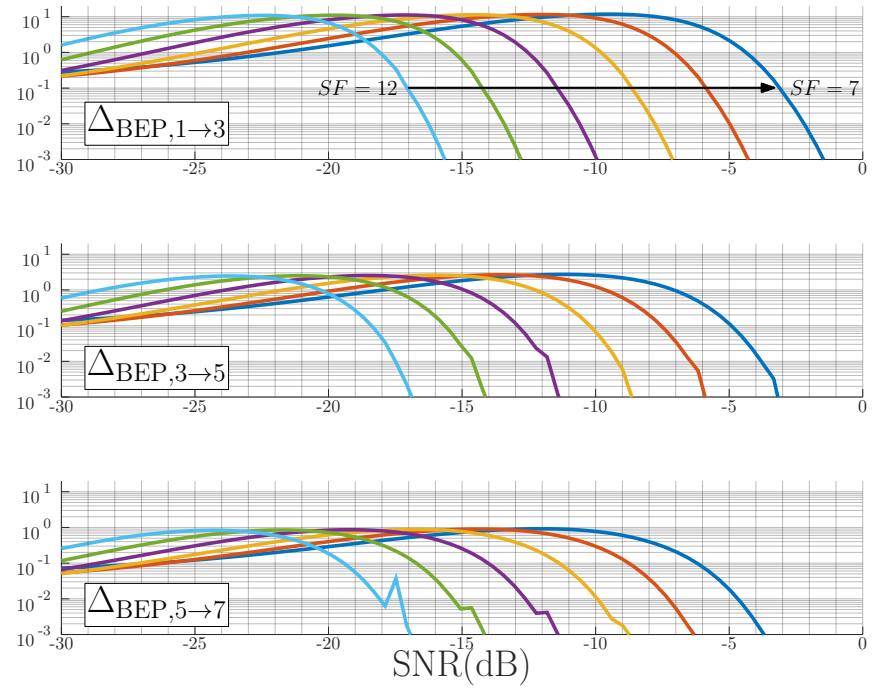

Fig. 4: Accuracy loss when increasing approximation order $\Delta_{\mathrm{BEP}, 1 \rightarrow 3}, \Delta_{\mathrm{BEP}, 3 \rightarrow 5}$, and $\Delta_{\mathrm{BEP}, 5 \rightarrow 7}$ as a function of SNR for $S F=7$ to 12 .

accurate, their contributions are less significant than the odd ones. It means that odd orders should be used, typically $\epsilon=3$.

\section{B. SNR-corrected zero-order approximation}

The complexity computation is linked to the number of Marcum functions in the formula, which is equal to $\epsilon+1$. It means that the lowest approximation, i.e. $\epsilon=1$, needs to compute two Marcum functions. In order to limit the complexity, we propose a heuristic zero order approximation, i.e. only one Marcum function, based on the case $\epsilon=1$ associating the same bound $z_{c}(1)$ and a correction $\gamma$ applied to the SNR:

$$
\mathrm{SEP}=1-Q_{1}(\sqrt{2 N \gamma \mathrm{SNR}}, \sqrt{2 \log (N-1)}) .
$$

A straightforward numerical study based on minimizing the mean square error was done in order to fit the zero-order expression to the 7 th-order one as a function of $S F$. The obtained numerical values are given in Table II and BEP curves are plotted in Fig. 5. The first observation is that the values of $\gamma$ depend on $S F$. A numerical approximation was found that gives the tractable form:

$$
\gamma \simeq \sqrt{2 S F} / 20+0.681
$$

The BEP comparison shows that the approximation is still close to the 7 th order. It is confirmed with the relative error

TABLE I: Numerical values for the maximum value of $\Delta_{\mathrm{BEP}, i \rightarrow i+1}$ when $S F=7$ to $12, i=1$ to 6

\begin{tabular}{|c|c|c|c|c|c|c|}
\hline$i$ & 1 & 2 & 3 & 4 & 5 & 6 \\
\hline $\max \Delta_{\mathrm{BEP}, i \rightarrow i+1}$ & $11.6 \%$ & $0.9 \%$ & $2.4 \%$ & $0.4 \%$ & $0.76 \%$ & $0.17 \%$ \\
\hline
\end{tabular}

TABLE II: Numerical values for SNR correcting coefficient

\begin{tabular}{|c|c|c|c|c|c|c|}
\hline$S F$ & 7 & 8 & 9 & 10 & 11 & 12 \\
\hline$\gamma$ & 0.868 & 0.882 & 0.894 & 0.905 & 0.915 & 0.924 \\
\hline
\end{tabular}

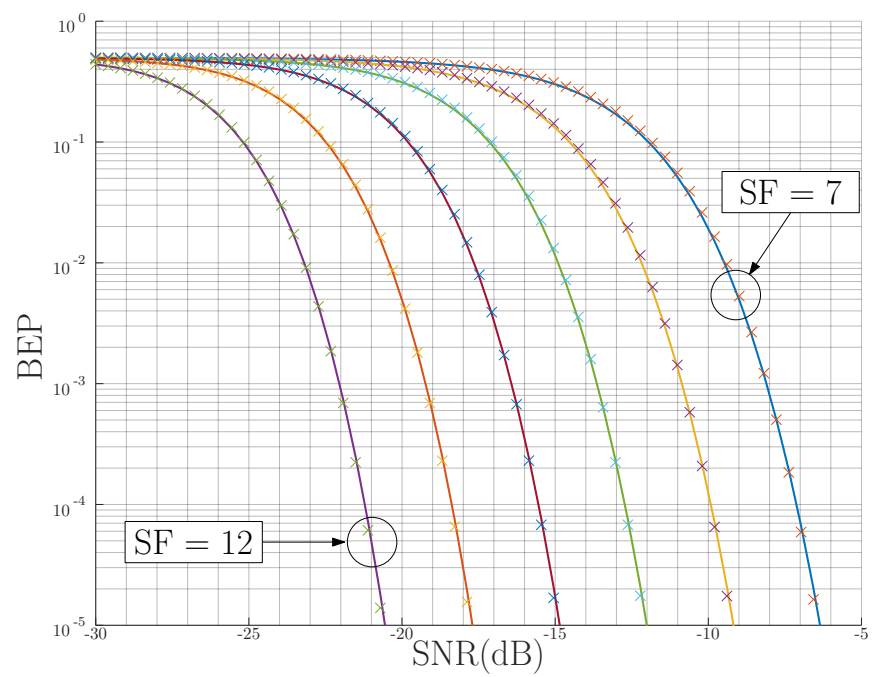

Fig. 5: BEP comparison of the SNR-corrected zero-order approximation (cross marquer) with the 7 th one for $S F$ equal to 7 to 12 (line).

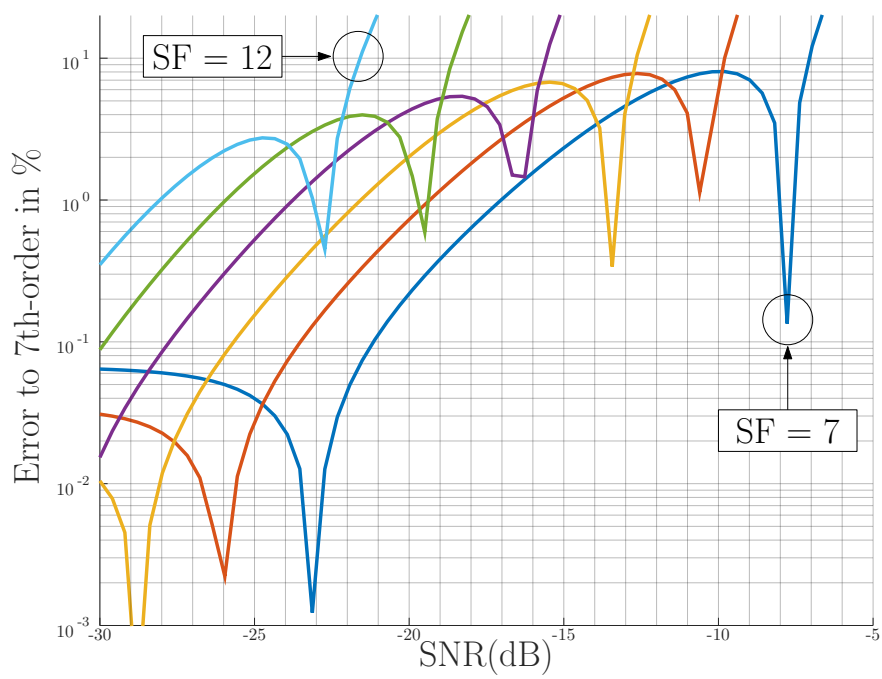

Fig. 6: Relative error of the SNR-corrected zero-order approximation with the 7th one for $S F$ equal to 7 to 12 .

plotted in the Fig. 6. The error is less than $10 \%$ but it increases very fast at high SNR: the diversity order is different and the values diverge. Moreover, since the error is plotted in absolute values, an inflexion point is visible that corresponds to the equality. In conclusion, the approximation is close to the exact value but is available for a given BEP range, i.e. a value of BEP inferior to $10^{-6}$ in our proposal.

\section{BEP comparisons}

Fig. 7 compares the proposed approximation with simulated BEP and the two results of [9] which are the best of the state of the art. These simulations validate that the Marcum-based approximation is tightly accurate with a low order $\epsilon=5$. At high SNR, our proposition and [9, eq.(21)] are equivalent and close to simulation but [9, eq.(23)] is less accurate. However, differences are visible at low SNR: both solutions of [9] 


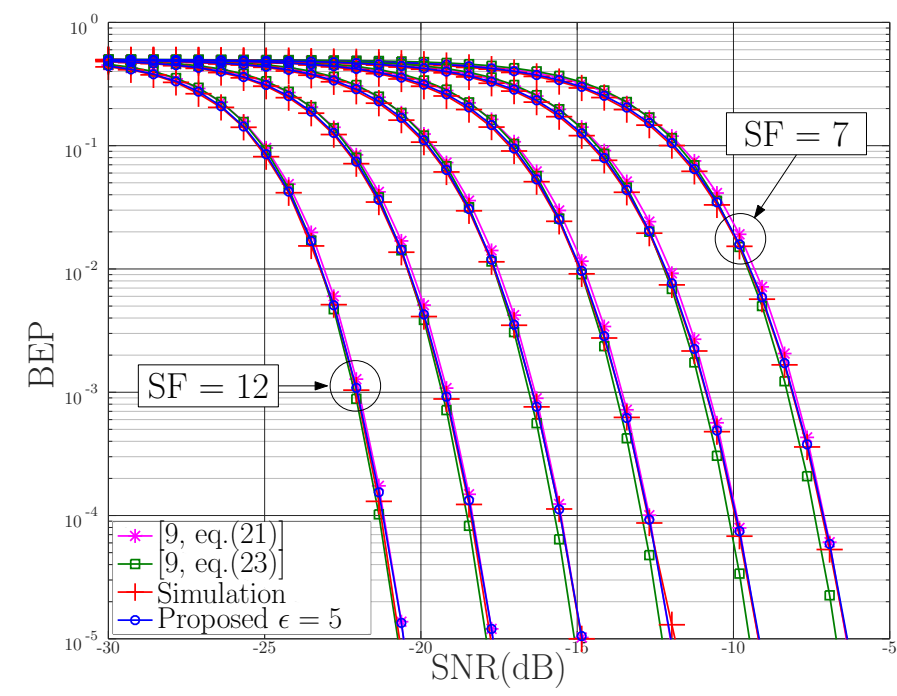

Fig. 7: Comparison of the proposed approximation with simulation and [9], the order $\epsilon$ is 5 and $S F=7$ to 12 .

are upper bounds while our approximation is still close to simulations. In order to highlight these differences, Fig. 8 proposes to focus on the relative error of approximations with respect to the simulated BEP. The approximations [9, eq.(21)] and [9, eq.(23)] are compared to our proposed solution with $\epsilon=0,3,5$. The observation of the curves confirms that the Marcum-based expression is more accurate than both [9, eq.(21)] and [9, eq.(23)] and increasing the order $\epsilon$ enhances the accuracy. At $\mathrm{SNR}=-15 \mathrm{~dB}$, the approximation error for a spreading factor of 7 is about $1 \%$, which represents a gain of almost 10 compare to previous works. On the other hand, the heuristic approximation for $\epsilon=0$ achieves an error quasiequivalent to $\epsilon=3$ and might propose the best trade-off complexity/accuracy.

\section{CONCLUSION}

In this paper, a new approximation of BEP for LoRa transmissions is proposed for AWGN channel. Instead of approximating a Ricean or Chi squared distribution thanks to a Gaussian one, we proposed to keep the noncentral Chi squared distribution, leading to a Marcum-based form. Simulation results confirm that the proposed solution is more accurate than the state-of-the-art for a full SNR range and may be considered as a numerical reference of the LoRa BEP for helping the design of a communication. On the other hand, a heuristic simplified version was derived in order to keep only one Marcum function to compute.

\section{ACKNOWLEDGMENT}

This work was supported by the ALAMO project labelled by the competitiveness Pole "Image and Networks" and founded by Région Bretagne and Lannion Trégor Communauté.

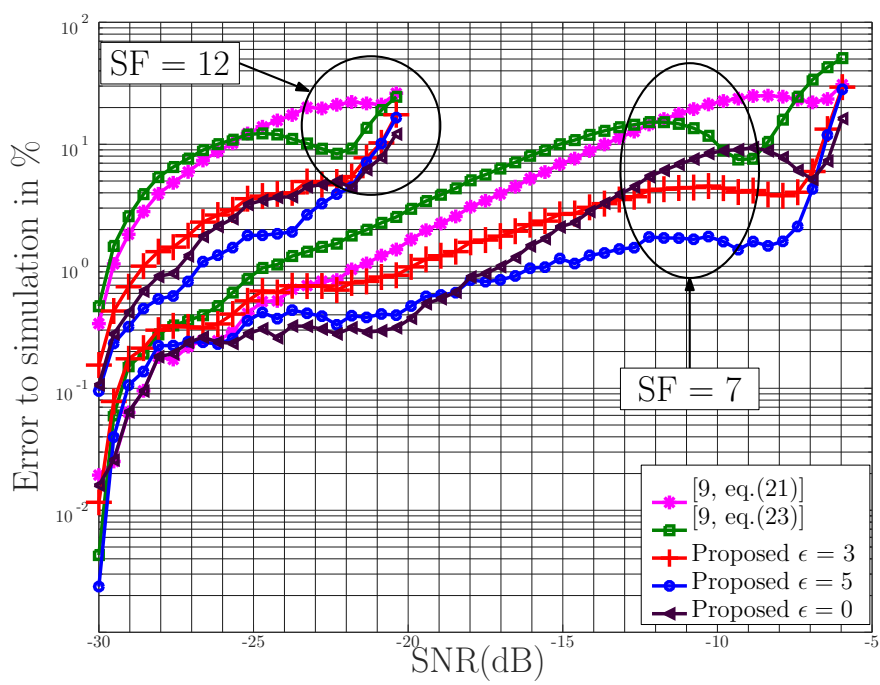

Fig. 8: Relative error between the proposed approximation and [9] with respect to the simulation for $\epsilon=0,3,5$ and $S F=7,12$.

\section{REFERENCES}

[1] F. Ait Aoudia, M. Gautier, M. Magno, M. Le Gentil, O. Berder, and L. Benini, "Long-Short Range Communication Network Leveraging LoRa and Wake-up Receiver," Microprocessors and Microsystems: Embedded Hardware Design (MICPRO), vol. 56, pp. 184 - 192, December 2017.

[2] R. Sanchez-Iborra and M. Cano, "State of the art in LP-WAN solutions for industrial IoT services," Sensors, vol. 16, no. 5, p. 708, 2016 [Online]. Available: https://doi.org/10.3390/s16050708

[3] O. Seller and N. Sornin, "Low power long range transmitter," Aug. 2014, US Patent App. 14/170,170. [Online]. Available: https://www.google.com/patents/US20140219329

[4] Semtech, "SX1272/73-860 MHz to $1020 \mathrm{MHz}$ low power long range transceiver," Tech. Rep. Datasheet, Mar. 2015. [Online]. Available: http://www.semtech.com/images/datasheet/sx1272.pdf

[5] A. Springer, W. Gugler, M. Huemer, L. Reindl, C. C. W. Ruppel, and R. Weigel, "Spread spectrum communications using chirp signals," in IEEE/AFCEA EUROCOMM 2000, Information Systems for Enhanced Public Safety and Security, 2000, pp. 166-170.

[6] B. Reynders and S. Pollin, "Chirp spread spectrum as a modulation technique for long range communication," in 2016 SCVT, Nov. 2016, pp. $1-5$.

[7] X. Le, B. Vrigneau, M. Gautier, M. Mabon, and O. Berder, "Energy/reliability trade-off of LoRa communications over fading channels," in 2018 25th International Conference on Telecommunications (ICT), June 2018, pp. 544-548.

[8] L. Vangelista, "Frequency shift chirp modulation: The lora modulation," IEEE Signal Processing Letters, vol. 24, no. 12, pp. 1818-1821, Dec 2017.

[9] T. Elshabrawy and J. Robert, "Closed form approximation of LoRa modulation BER performance," IEEE Communications Letters, vol. 22, no. 9, pp. 1778-1781, 2018.

[10] J. G. Proakis, Digital Communications, 5th edition, N. York:McGrawHill, Ed., 1995.

[11] J. I. Marcum, Table of Q functions, R. Corporation, Ed., Jan. 1950.

[12] M. K. Simon and M. . Alouini, "Exponential-type bounds on the generalized Marcum Q-function with application to error probability analysis over fading channels," IEEE Transactions on Communications, vol. 48, no. 3, pp. 359-366, March 2000.

[13] B. Reynders, W. Meert, and S. Pollin, "Range and coexistence analysis of long range unlicensed communication," in The 23rd International Conference on Telecommunications (ICT), May 2016, pp. 1-6. 